\section{A sparkling clean success story}

DAC Universal has stood for excellent hygiene and safety standards for 20 years. A remarkable characteristic in the field of dental solutions - as remarkable as its mode of operation. DAC Universal does not require chemical cleaning agents but uses only demineralised water for the reprocessing of medical instruments - a feature that helps to preserve the lifespan of instruments. Cleaning, thermal disinfection or unwrapped sterilisation and optional lubrication: the fully compliant and automatic reprocessing cycle takes only 15 or 21 minutes, depending on the programme. All cleaning steps are standardised and fully automated at the touch of a button, which significantly minimises application errors.

In 2000, the launch of the first DAC Universal changed reprocessing in dental clinics. Five years later, the first updated edition of DAC Universal entered the market, followed by a new model again in 2009. By 2017, 40,000 DAC Universal units had been installed worldwide. Over the years, many technical improvements and upgrades

\section{Specialist dental accountants with a new name}

Figurit are specialist dental accountants that offer full-service accountancy, tax, bookkeeping and advice.

Figurit was formerly known as Lansdell \& Rose. For 25 years, this was a name synonymous with quality tax planning and financial guidance, helping dental and medical professionals get the most out of their business.

Nothing has changed except the name!

The refresh will create a similar brand to Figurit's sister company Wagemate, a payroll service that operates throughout the UK, www.wagemate.com.

Figurit will help you optimise your personal finance too. For all your accountancy needs, contact the team today.

For more information visit https:// figurit.com or call Figurit (formerly known as Lansdell \& Rose) on 0207376 9333. have been added, including the graphic display, intuitive user interface, and the possibility to reprocess not only contra-angle handpieces and turbines, but also ultrasonic/ sonic handpieces and tips as well as nozzles of multifunctional syringes, and powder jet devices and handpieces. Today, DAC Universal meets the expectations of dental professionals, offering professional care, cost-effective reprocessing, fully automatic reprocessing, and legal certainty.

The latest update includes new features that enhance user-friendly handling: touch display, LAN interface, guided Check \& Clean maintenance workflow.

DAC Universal D, launched in 2019, features an intuitive user interface with its touch display. In approximately 15 minutes, up to six instruments are reprocessed fully automatically at the touch of a button.

Launched in September, the new DAC

Universal S completes the DAC Universal product portfolio with a sterilisation function especially for markets that require sterilisation instead of a disinfection of unwrapped medical devices.

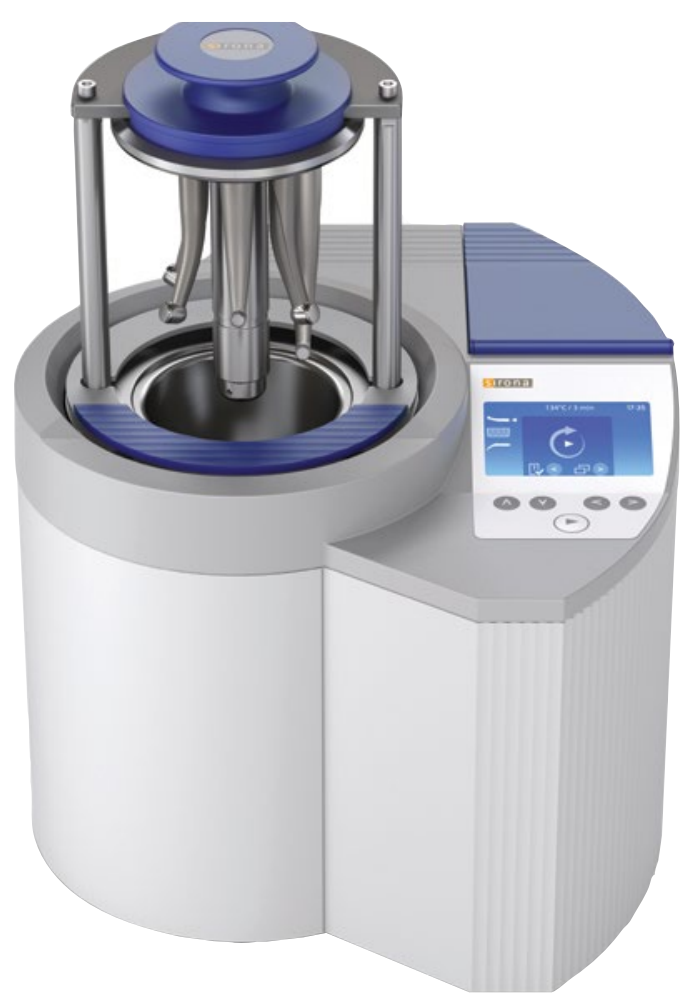

Find out more about the DAC Universal Infection Control Systems at https://www. dentsplysirona.com/en/explore/infectioncontrol-systems/dac-universal.html.

\title{
A broad range of indications
}

\section{BRILLIANT Crios is a} reinforced composite bloc, part of COLTENE's proven, high-quality range used for forward-thinking dentists.

It can be used for restorations without a separate firing process. It has excellent mechanical properties and, with two translucencies and 13 shades, your results will be as natural and beautiful as they are functional.

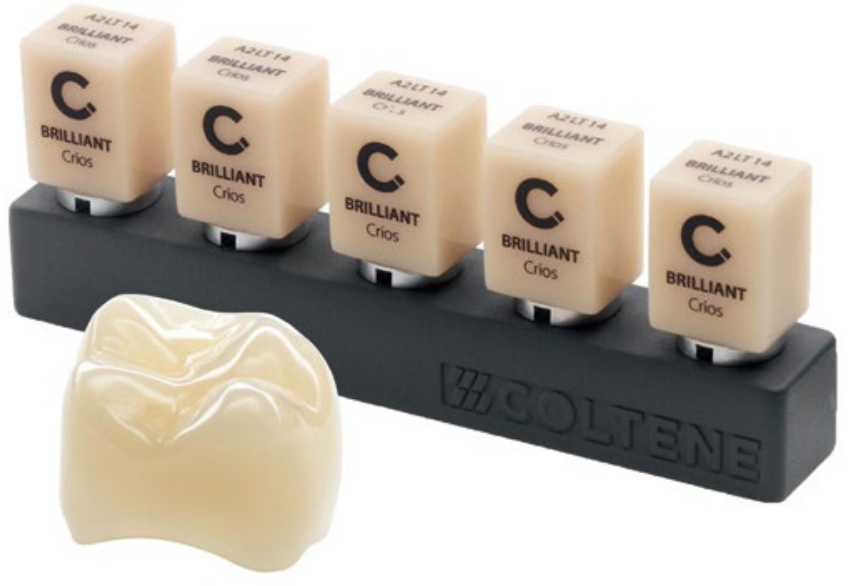

ideal for:

- Single tooth restorations, anterior and posterior

- Inlays, onlays, crowns and veneers

- Bruxism patients and implant-supported crowns - the material is a great shock absorber.

For efficiency, aesthetics and value, upgrade your results with BRILLIANT Crios - the all-rounder for everyday brilliance.

For more on COLTENE, visit www.coltene.com, email info.uk@coltene.com or call 0800 2545115 